\title{
25
}

\section{Sommerrätsel (Suchrätsel)}

Finde alle unten stehenden Begriffe im Suchrätsel. Die verbleibenden Buchstaben ergeben (Zeile für Zeile gelesen) das elfstellige Lösungswort.

Hinweis Die Begriffe sind von links nach rechts oder von oben nach unten zu lesen - nie diagonal oder rückwärts.

ART - AST - CILIE - COR - FORELLE - GIFT - HAMMER HAND - ION - IRIS - KOMPLEXAUGE - MIKROSKOP MORGENTAU - MYELIN - NEOPHYTA - PFAU - PHEROMON SAEUGETIER - SINNESORGAN - ZUNGE

\begin{tabular}{|c|c|c|c|c|c|c|c|c|c|c|}
\hline M & I & K & R & O & S & K & O & P & S & O \\
\hline S & I & N & N & E & S & O & R & G & A & N \\
\hline A & R & M & E & H & A & M & M & E & R & P \\
\hline E & I & F & O & M & E & P & F & A & U & H \\
\hline U & S & O & P & C & I & L & I & E & R & E \\
\hline G & A & R & H & M & Y & E & L & I & N & R \\
\hline E & C & E & Y & A & B & X & A & S & T & O \\
\hline T & O & L & T & R & H & A & N & D & E & M \\
\hline I & R & L & A & T & Z & U & N & G & E & O \\
\hline E & N & E & I & O & N & G & I & F & T & N \\
\hline R & D & M & O & R & G & E & N & T & A & U \\
\hline
\end{tabular}

Lösung:

(C) Springer-Verlag Berlin Heidelberg 2016

C. Reinbold, Fetthenne, Moderlieschen, Warzenbeißer,

DOI 10.1007/978-3-662-52817-4_25 